\title{
Health 4.0: Applications, Management, Technologies and Review
}

\author{
Type of article: Review \\ Ana Carolina Borges Monteiro1, Reinaldo Padilha França1, Vania V. Estrela2, Yuzo lano1, \\ Abdeldjalil Khelassi3, Navid Razmjooy4 \\ 1State University of Campinas (UNICAMP), Brazil, \{monteiro@decom.fee.unicamp.br, \\ reinaldopadilha@msn.com, yuzo@decom.fee.unicamp.br \} \\ 2 Universidade Federal Fluminense, Rio de Janeiro, Brazil, vania.estrela.phd@ieee.org \\ 3 University of Tlemcen, Algeria \{ khelassi.a@gmail.com \} \\ 4 Department of Electrical and Control Engineering, Tafresh University, Tafresh 39518 79611, Iran \\ \{ navid.razmjooy@ieee.org \}
}

\section{Abstract}

The Industry 4.0 Standard (I4S) employs technologies for automation and data exchange through cloud computing, Big Data (BD), Internet of Things (IoT), forms of wireless Internet, $5 \mathrm{G}$ technologies, cryptography, the use of semantic database (DB) design, Augmented Reality (AR) and Content-Based Image Retrieval (CBIR). Its healthcare extension is the socalled Health 4.0.

This study informs about Health 4.0 and its potential to extend, virtualize and enable new healthcare-related processes (e.g., home care, finitude medicine, and personalized/remotely triggered pharmaceutical treatments) and transform them into services.

In the future, these services will be able to virtualize multiple levels of care, connect devices, and move to Personalized Medicine (PM). The Health 4.0 Cyber-Physical System (HCPS) contains several types of computers, communications, storage, interfaces, biosensors, and bio-actuators. The HCPS paradigm permits observing processes from the real world, as well as monitoring patients before, during, and after surgical procedures using biosensors. Besides, HCPSs contain bio-actuators that accomplish the intended interventions along with other novel strategies to deploy PM. A biosensor detects some critical outer and inner patient conditions and sends these signals to a Decision-Making Unit (DMU). Mobile devices and wearables are present examples of gadgets containing biosensors. Once the DMU receives signals, they can be compared to the patient's medical history and, depending on the protocols, a set of measures to handle a given situation will follow. The part responsible for the implementation of the automated mitigation actions are the bio-actuators, which can vary from a buzzer to the remote-controlled release of some elements in a capsule inside the patient's body.

Decentralizing health services is a challenge for the creation of health-related applications. Together, CBIR systems can enable access to information from multimedia and multimodality images, which can aid in patient diagnosis and medical decision-making.

Currently, the National Health Service addresses the application of communication tools to patients and medical teams to intensify the transfer of treatments from the hospital to the home, without disruption in outpatient services.

HCPS technologies share tools with remote servers, allowing data embedding and BD analysis and permit easy integration of healthcare professionals' expertise with intelligent devices. However, it is undeniable the need for improvements, multidisciplinary discussions, strong laws/protocols, inventories about the impact of novel techniques on patients/caregivers as well as rigorous tests of accuracy until reaching the level of automating any medical care technological initiative.

Keywords: Pervasive healthcare, Wireless communications, Cyber-physical Systems, mHealth, Hospital Information System (HIS), Smart pharmaceuticals, Medical databases

Corresponding author: Vania V. Estrela, Universidade Federal Fluminense, Rio de Janeiro, Brazil, vania.estrela.phd@ieee.org

Received: 31 December, 2018, Accepted: 02 January, 2019, English editing: 04 January, 2019,Published: 05 January, 2019.

Screened by iThenticate..๑2017-2019 KNOWLEDGE KINGDOM PUBLISHING.

\section{Introduction}

The Internet of things (IoT) entails sets of gadgets, vehicles, and home equipment that contain hardware, programming, actuators, and network support, which enables to interface and trade data. Hence, these devices can 
impart and join forces over the Internet possibly using remote observation and control.

The Internet of Services (IoS) paradigm can connect gadgets intelligently. Makers need to thoroughly consider their business models to meet their expectations properly with a long haul income stream. Numerous producers may perceive this and exploit the chance to enhance their activities. The individualization of large-scale manufacturing and the IoSs include extra income. The savvy plant should be adaptable and convey intelligent items. A noteworthy misconception is not a cost sparing activity. Instead, it is another business model to expand income and gainfulness.

The (IoT), the IoS and so forth can comply to the Industry 4.0 standard [11] since it allows for the physical processes' virtualization and their transformation into services $[4,5,45]$ having in mind for the health domain that things such as artificial organs, biosensors, smart devices [20], and smart pharmaceuticals are already available. Hereafter, services will turn around these objects to virtualize several levels of care, help patients and healthcare professionals to reach independence, link up devices and technologies, and move towards the personalized medicine $[5,6]$.

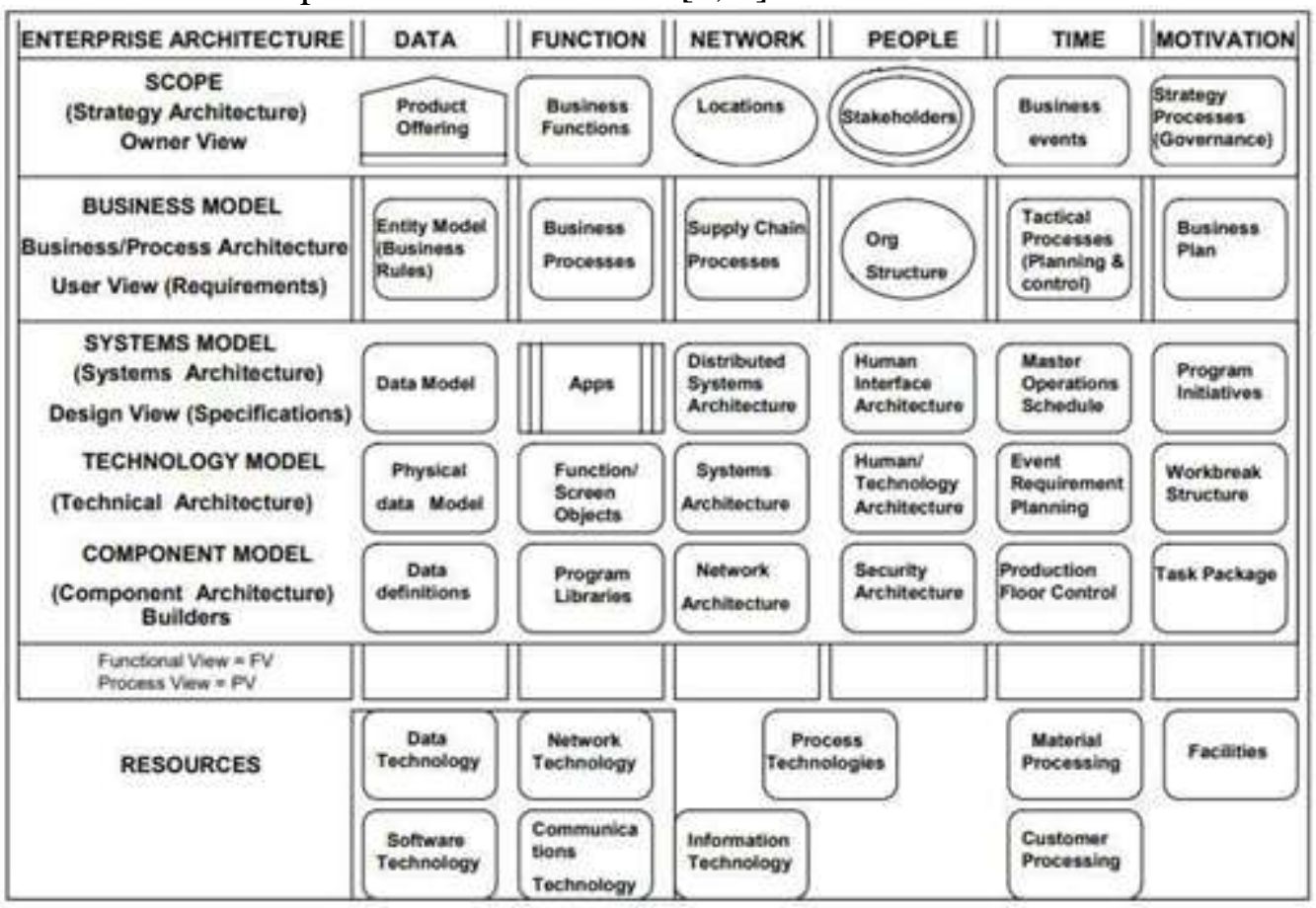

Figure 1. Zachman framework and the value chain reference model $[6,7,8,9,10]$

Smart Industry 4.0 compliant plants are context-aware and assist people with equipment to execute tasks. The term context-aware means the system can consider context data, for instance, the QR code, position, and status of an item. These systems depend on data to conclude their real life and virtual tasks. Real world evidence, e.g., temperature, position, operation time and condition of an instrument, in contrast to virtual data, like e-documents, multimedia content, and simulation results $[1,6,7,8,9,10]$. Clinics and distributed healthcare providing arrangements such as General Practice (GP) networks, public nurses, pharmacies, and so on being similar to factories, to facilitate context-aware people assistance and the correct use of machines during their duties, which can happen in Hospital Information Systems (HIS) 
or practices IT systems. Existing flaws in healthcare facilities and settings are frequently related to real-time information since it is limited and that makes workflows difficult to be depicted accurately. Sometimes, the patient or expert location or her/his current status are not known. The regular fall-out can be a disruption of the operating schedules with the medical staff waiting for the patient in the operating room or if patients wait for extended times in Accident \& Emergency (A\&E) and outpatient sectors. Smart medical plants need to incorporate some of the practices from the industrial domains.

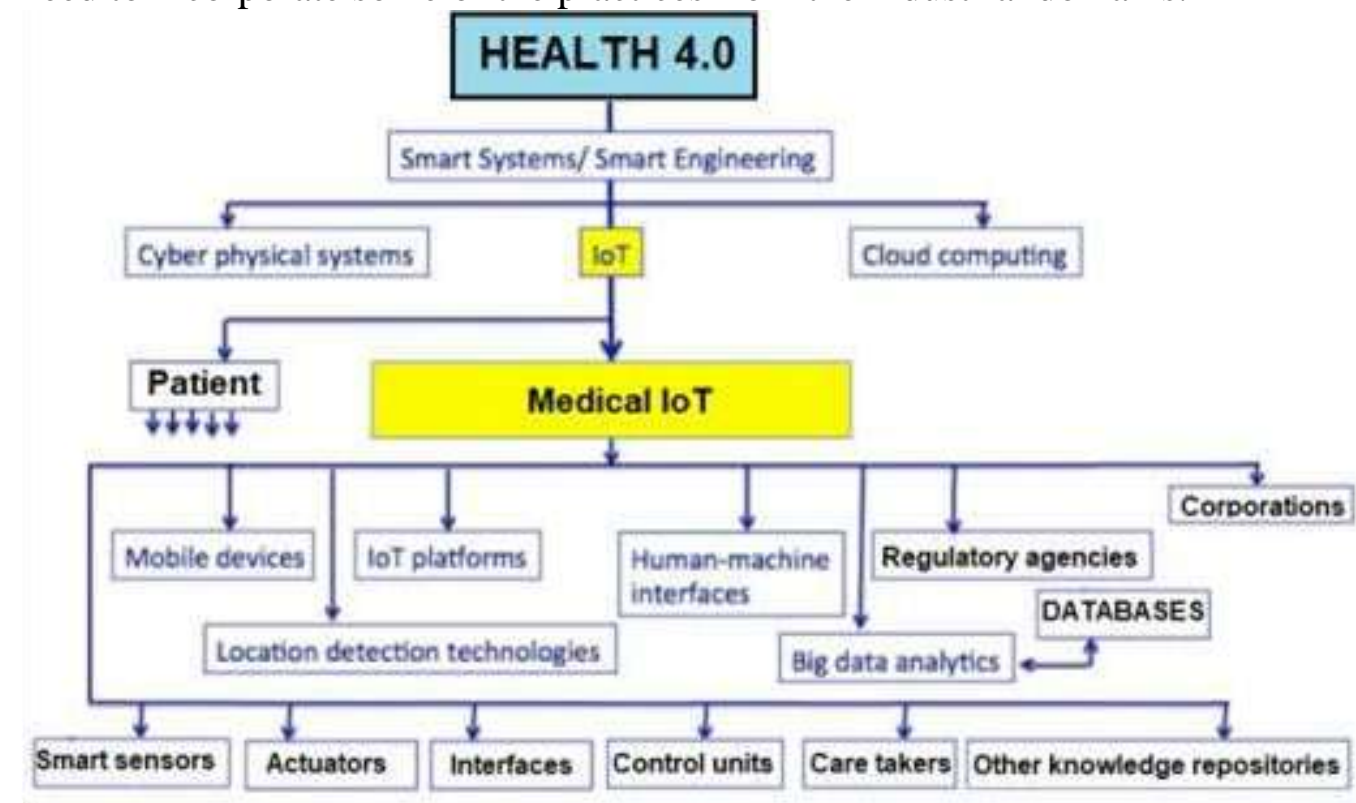

Figure 2. The proposed framework for the Health 4.0

\section{Industry 4.0 Scalability into the Health Domain}

Through the Industry 4.0, a Cyber-Physical System (CPS) can monitor realworld processes, producing corresponding a virtual rendition of the setting and implement decision-making in a decentralized fashion. Over the IoT, a CPS converses and cooperates with humans in real-time. Likewise, the IoS offers both inner and cross-organizational services for the value chain partakers $[1,2,3,4,5,6,7,10,33]$.

Value Chain (VC) Organization (VCO) is paramount to healthcare facilities to boost their usefulness and productivity in the presence of budget pressures (Figure 1). VCO involves the activities within the organizational boundaries form the VC, which, in turn, forms parts of the supply chain while linking suppliers with customers $[6,7,10]$.

Both the flow and the pathways for patients are the traditional VCs' paradigms within the healthcare business, which resemble any other business concerning VCs and can benefit from the Industry 4.0 standard. Healthcare will undoubtedly be organized modularly as specialization increases, and the global healthcare model is gradually shifting from a hospital-based professional-oriented to a distributed patient-centered healthcare model [8]. A distributed patient-centered healthcare system $[34,35,36]$ must have its elements and services available to the health-deprived subject and the associated caretakers. CPSs still need popularization in the medicinal domain, but the process has started. Pharmaceutical corporations are developing smart biosensors and pharmaceuticals to enable real and virtual worlds communications. Big Data (BD) strategies will be responsible for 
individualization and custom-made healthcare. Novel strategies such as precision medicine relied on real-time connectivity concerning real-world patients, cloud-based procedures and virtually deployed autonomous systems. These tactics will combine cross-organizational services depending heavily on real-time information demanding new health supervision models demand individual patient budgets offering to patients and informal caretakers more impact and control of their health and the pertinent resources at their disposal [9].

\section{Industry 4.0 Design Principles}

According to [1], Industry 4.0 design principles are the following:

- Interoperability;

- Virtualization;

- Decentralization;

- Real-time capability;

- Service orientation; and

- Modularity.

Interoperability simplifies the contextual information flow on all levels. Observing the biosensors as part of CPSs and their backend in the virtual domain smooth interoperability is essential to enable the whole system loop to accomplish and continuously exchange data. In CPSs, it is also vital to have different services combined and integrated to significantly improve data readings to guarantee creation and recording of meaningful data. Thus, interoperability arises as a fundamental design principle of Health 4.0 solutions [34, 35, 36].

Virtualization must be available, but some issues must be addressed. A CPS can monitor the physical processes while creating a virtual copy of the reality at a given time. Smart factories have virtual models that include the condition of all other CPSs. Almost certainly; these trends are usable for the health domain in several ways. The observing physical processes are the key to deploy health-related processes on a daily basis. CPSs will be monitoring patients through surgical procedures extensively in a standardized fashion everywhere [19]. Nonetheless, the patients' biosensors during surgery can still benefit from novel solutions. One key problem is that generally; these islands are equivalent to closed-loop systems. Hence, they cannot be easily connected to other systems, e.g., the Picture Archiving and Communication System (PACS). Moreover, thanks to the human beings' multifariousness as a system, to copy of the entire reality periodically is unfeasible. However, a valid question is undoubtedly in how far this is sensible and necessary. The monitoring and virtualization of defined system sections might be sufficient until future technologies allow for more wide-ranging and easier virtualization. Currently, the challenge in the healthcare domain is that autonomous virtualization happens anywhere, anyway and whenever needed. This is particularly interesting to new strategies for the individualization of therapies (mainly to treat long-lasting, non-communicable illnesses [12]) 


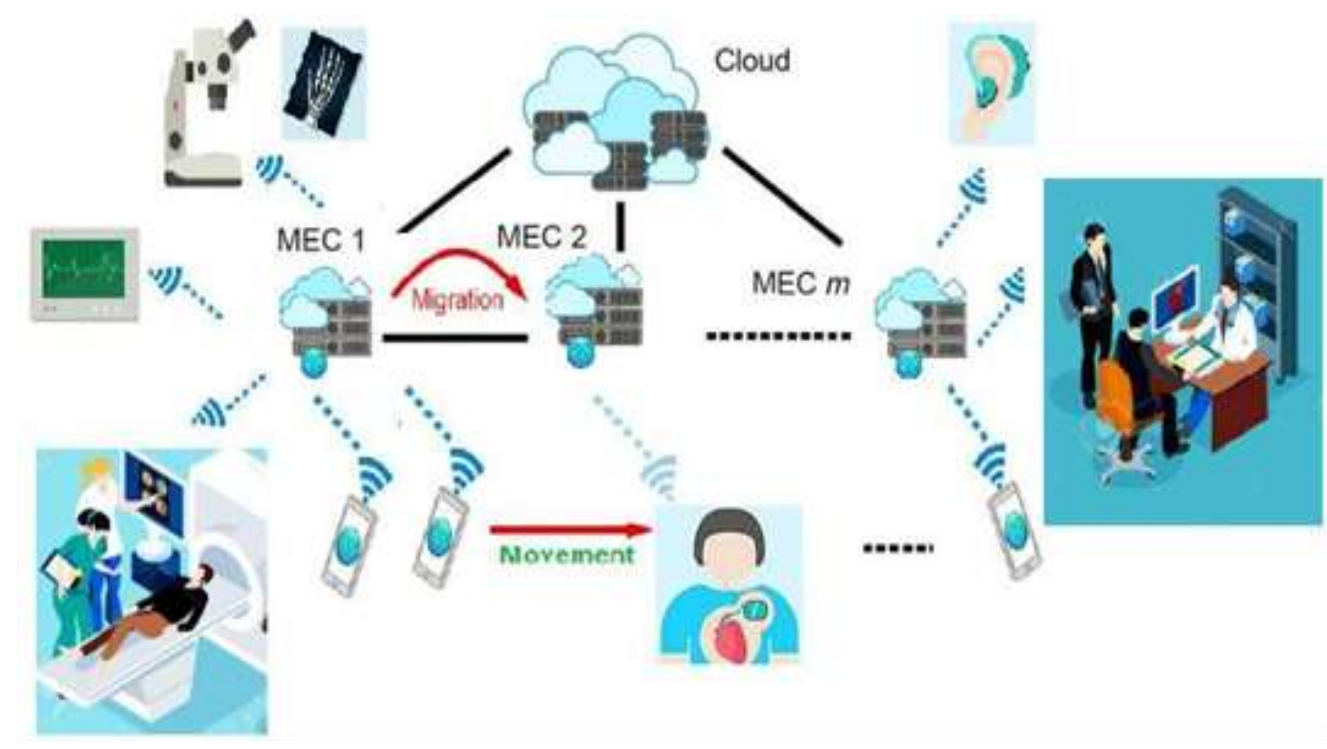

Figure 3. Mobile Edge Cloud MEC in the healthcare domain [22]

Healthcare decentralization is generally considered as challenging since it does give sufficient acclaim and, consequently, it does not appeal to most underdeveloped nations. Otherwise, progressively more patients will have GP surgeries, day treatment centers, their households, and over the Internet. Still, as more smart devices, wearables, and bio-actuators are sold for both fitness and welfare, these gadgets' accuracy and appropriateness are questionable. Governance and liability matters are still pendent. The estimated amount of applications for healthcare, wellness, and beauty on the market is high, which calls for more rigorous testing of equipment and apps. Concerning accuracy, even fewer give warranties. Although healthcare is leaning toward a distributed patient-centered model with patients, specialists and formal and informal caretakers progressively using smart devices [20], biosensors, bioactuators, apps, and CPSs, ever more sophisticated needs are building up about the network and communications providers. Distributed patientcentered care requires a continuous and reliable data flow across diverse networks and spheres. The refined necessities of various domains comprising healthcare have led to numerous research works $[16,17]$. The National Health Service (NHS) addresses a strategy to apply information communication tools for patients, their caretakers and medical staff to intensify the number of treatments transfers from hospital to homecare without disturbing their outpatient services too much [18].

The use of technology like barcode and Radio-Frequency Identification (RFID) in Industry 4.0 plants can help autonomous decision-making as happens in the NHS and other countrywide healthcare services [19, 20]. An additional compelling aspect is intelligence and processing deployment into networks with distributed parts. The Mobile Edge Cloud (MEC) computing arrangements are more than a tendency [22] (Figure 3). It is an effort to support decentralized network policymaking to diminish latency and augment security. MEC is a popular subject among network technology providers due to a rising trend towards decentralization in the healthcare realm, then a robust technology to deploy the Industry 4.0 design principles. This growth is vital to release efficiency assets in healthcare and meet future socio-economic requirements [22].

The real-time functionality can be paramount for any organization involving persons, notwithstanding the domain to safeguard and maintain performance. 
Part of the customization rationale is the real-time identification of people's necessities in a distributed fashion. Patients should receive wherever possible treatment outside hospitals with the exact amount of medication to maximize therapeutic results while lessening secondary effects. This is known as theragnostics [18] where (ideally) diagnostics, control, relief, and remediation merge and approach real-time [21] to form a harmonious spatiotemporal entity. This functionality is crucial to healthcare and it will lead to the implementation of personalized prescriptions, smart medications, intelligent bio-actuators' use, and supply chain administration.

Service Orientation (SO) in healthcare organizations are shifting to a customer-SO with reactive groups waiting on the platform. A high-level overview of customer-centered service aggregation of the IoT, the IoS, the IoP and so forth combined with customized services [1, 11] also work for healthcare where SO can be part of the Health 4.0 standard [11, 19]. Likewise, pharmaceutical industries tend to shift from being only medications manufacturers to become healthcare service providers. The underlying rationale is to harvest $\mathrm{BD}$ from a colossal variety of smart devices, biosensors and bio-actuators to work with smart devices, biosensors, and bio-actuators to prevent any harm and serious episodes, lessen sick days in addition to hospital admission. These policies will expand the life quality and decrease associated prices and dependencies. This means that a health-related company will sell disease administration procedures as services from a business model viewpoint. In contrast, healthcare suppliers may soon only receive products that exceed the plain delivery of treatment or medications. Patients' healthcare records deliverance via a distinct interface may become an admission prerequisite to access some healthcare. Patients can sanction the use of their healthcare data as a service and sell this information to healthcare establishments to speed up trials (in the case of ill-doing) or the creation of new lines of disease attack. All these current speculations and the forthcoming $5 \mathrm{G}$ technology will enhance the healthcare domain SO. Eventually, both the network slice expertise, the MEC technologies, and possibly novel developments will help to set up service aggregation through different realms and networks [22].

Modularity in healthcare is important because a modular system can adjust to changing settings and requirements while replacing or expanding subsystems. Consequently, modular systems can be effortlessly adjusted to handle seasonal fluctuations or changes in products/systems characteristics. [22, 38, 39]. Modular software components must engender new functionalities by just recombining different active groups. Enforcing the rules to reflect norms and standards alongside the system's software modules is an effective approach to program faster and instituting new functionalities from predefined software building blocks [23]. Users may consult and evaluate a collection of services and characteristics to choose products that fulfill their needs and wants [24, 37].

From the healthcare domain viewpoint, the security dimension is worth commenting [1] since it is a critical infrastructure for any nation that calls for protection of all the interrelated functionalities and the confidentiality of the personal data. These delicate matters differ somehow from a smart factory where security fissures may cause monetary losses or material damage without huge liability for personal data or loss of lives. Issues such as safety, robustness, confidentiality, and resilience while sparing all participants from 
incalculable and random risk are hard to design principles from a Health 4.0 angle. Trust is both a healthcare pivotal principle and a legal obligation addressed by most national legislation. The Health 4.0 domain needs to prioritize safety, robustness, and resilience as a general because in the Industry 4.0 these topics are basic requirements but do not tackle issues so delicate concerning human matters as health is stated by the WHO. A possibility is to extend the Industry 4.0 design criteria to the health domain [1].

\section{Health 4.0}

Health 4.0 is a tactical deployment, and managerial model for healthcare inspired by the Industry 4.0. Health 4.0 has to allow gradual virtualization to support the healthcare personalization close to real-time for patients, workers, and both formal and informal caretakers. This healthcare personalization calls for the substantial usage of CPSs, cloud computing, the extended specialized IoTs aka Internet of Everything (IoE) including appliances, services, people, and surfacing 5G communication networks. With the help of the CPS paradigm, software fit for distributed systems and BD tools, algorithms, and objects will be virtualized employing a spatial-temporal matrix. The virtualization permits the inspections of small space-time windows of the real world in real-time and, thus, allows for theragnostics $[12,18]$ in personalized and precise medicine.

\subsection{Improving Health Services through CPS Adherence}

The improvement of medical analyses must comply with the Industry 4.0 protocol with long-lasting behavioral modifications. These objectives must incorporate technologies like 5G, IoT, Narrow Band IoT (NB-IOT [12, 13]), network slices [13], cloud computing, Big Data (BD), and cryptography/security into real-time CPSs.

Some technology alternatives involve the use of embedded biosensors and bio-actuators. At first, smartphones were used as back-end devices to save data and deliver processing intelligence by creating connections to healthcare professionals. Smartphones can function as gateways to share information with remote servers hence enabling incorporation of data and BD analysis. Although smartphones can work like computers and potential gateways, there are some questions concerning reliability, appropriateness, and practicality. Characteristically, patients require different sorts of medications and medical equipment. Many patients stock several items in different places can be reached readily in case of need. This means that many medical types of equipment would be connected to the smart device permanently, which demands battery life and routers. Another challenge is the conflict regarding the reliability between mobile and medical devices as a whole. Although mobile devices normally function on a best effort basis, medical devices can be mission critical. The concepts of best effort and mission-critical task comprise the Quality of Service (QoS) but they mean different things. The best effort concept decides if sensitive patients' data will be sent, while the term mission critical guarantees the reliability of equipment, organization or 
process. Evidently, to guarantee a high Quality of Care (QoC) along with superior QoS and Quality of Experience (QoE) of medical devices, they should not work on a best effort basis since this can jeopardize the key objectives of a therapy, i.e., maximizing adherence [21] while minimizing the incidence of severe symptoms, attacks, hospitalization, and even death. Other significant aspects comprise energy efficiency and data protection and confidentiality at all levels. Exhausting mobile devices and wearables as gateways can improve energy efficiency but using smart devices while accessing the radio network is demeaning to energy efficient. Security communication links' and smart devices' biosensors' and actuators' signals need to be safe while complying with the standards and legal difficulties from the healthcare field [19].

Unusual technologies can expressively improve the current communication links for smart pharmaceuticals, intelligent treatments of varied forms and smart healthcare deployment strategies. NB-IOT requires a Low Power Wide Area (LPWA) to allow robustness to power shortages with all sorts of electromagnetic frequencies including Radio Access Technology (RAT). Smart power meters have employed similar knowhow to gauge households' consumptions more accurately. Still, this does not exclude smart devices, biosensors, and bio-actuators of being part of individualized therapy sometimes. Patients can manage their data and get therapy recommendations with their smart devices through video downloads for instance. They can also permit and follow up their information usage by healthcare professionals, caretakers, and researchers.

NB-IOT modules are an exciting and feasible solution to enhance connectivity by exploiting different segments of the electromagnetic spectrum in contrast to smartphones. In general, NB-IOT employs considerably lower frequencies than smartphones. Low-frequency waves have superior penetration and reach properties owing to their physical properties. However, the data bulk and the bandwidth are limited by the energy efficiency of the smart gadgets. The issue is if a medication cost increase can be justified through the value aggregated when using Industry 4.0 characteristics, such as CPS capability, modularity (to allow for several biosensors, bio-actuators and medications pertaining to a person to interact simultaneously), SO and interoperability (which means that services and CPSs may be combined to attain better disease control). Fundamental requirements for Health 4.0 are:

- Predictable QoS;

- Network agnostic and interoperable technology;

- Safe, robust, privacy-protected, and resilient technology;

- Complete connectivity and compatibility; and

- Worldwide product/service interoperability as well as network capabilities for a global service organization.

\subsection{Medical Internet of Things (mloT)}

Lack of knowledge about a health problem and the corresponding proper management can aggravate conditions and result in high mortality. The successful application of mIoT in diseases' management and health education are essential issues. With mIoT and 5G, all kinds of multimedia material related to disease education can be sent to the patients' mobile terminals, augmenting their knowledge about their conditions while integrating 
pharmacological and non-pharmaceutical treatments. In addition, mIoT facilitates the assessment and monitoring of illnesses. For example, patients can control their tests and questionnaires using cell phones habitually, so that doctors can observe their patients' states regularly. Alternatively, health specialists, decision-makers, and service providers can apply the mIoT to assess conditions dynamically and how they interact with environmental or behavioral aspects $[13,14,15,16,17,28]$.

\subsection{Databases}

The advent of Content-Based Image Retrieval (CBIR), Content-Based Video Retrieval (CBVR) and Content-Based Multimedia Retrieval (CBMR) systems have countless applications in medical settings. This manuscript will refer to them as Content-Based Retrieval (CBR) systems.

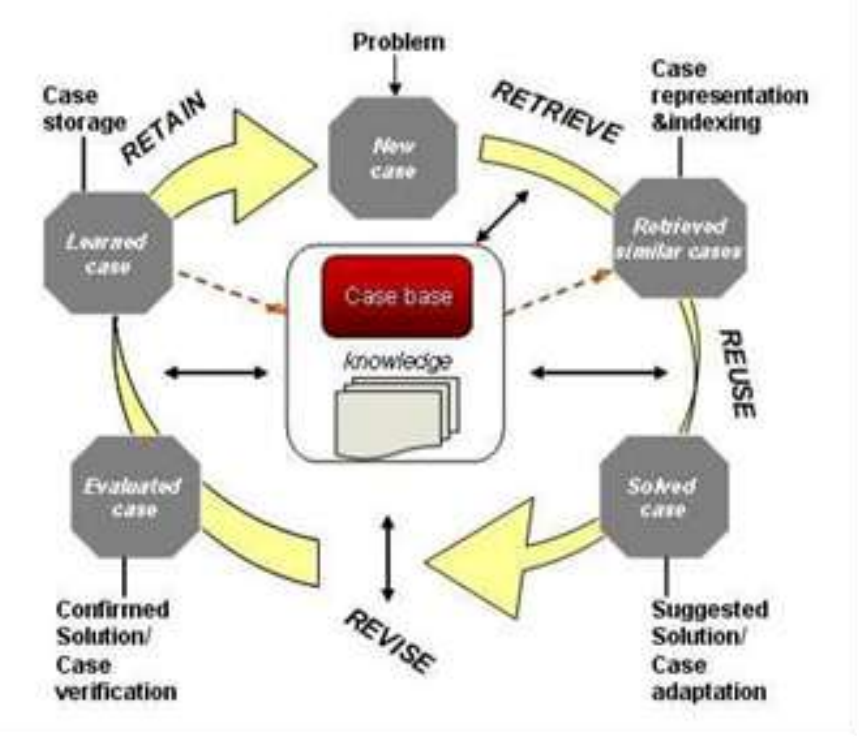

Figure 4. CBIR and CBVR in health care [24, 25]

A CBIR accesses information from still images and this way of diagnosing patients allows for an intuitive case representation for physicians and healthcare staff. The corresponding information can be structured by cases, which leads to a suitable way the semantic gap can be solved through medical semantics and semiotics knowledge and structured databases. Moreover, the multimedia data comprehension occurs incrementally in a CBR system. Besides these CBR benefits, there exist Translational Incremental SimilarityBased Reasoning (TISBR) structures [23] where the reasoning relies on the combined characteristics of different types of CBR systems containing information obtained with several modalities. A TISBR can provide incremental medical knowledge learning, collections of structured CBR cases, the multimedia usage of similar cases, and more information about different concepts of similarity. These objectives can be accomplished by means of the indexing, the use of cases retrieval, and the search refinement strategies $[24,25]$.

Better databases will also allow healthcare professionals to build more intelligence representations of the data with more information per pixel or voxel as well as high-resolution models with the help of deep learning [28, $29,30,31,32]$. 


\subsection{G-Driven Personalized Care}

The health treatments use different tools and procedures that need to comply with the commercially available smart devices, biosensors, actuators and wearables connected to medical CPSs. Furthermore, schemes adequate to custom-made medicine need to be devised. The latest generation of smart gadgets, biosensor, and bio-actuators technologies supports some surveillance of important treatment Key Performance Indicators (KPIs) at the healthcare points. KPIs like adherence [21], physiological parameters, and 5G timing will afford multi-frequency connectivity with multi-modal capability comprising NB-IoT and mobile communication to enable the real and the virtual realms to exchange information. This will facilitate theragnostics procedures and the smooth combination of several types of therapies $[12,26$, $27,33]$.

Theranostics is a treatment approach that associates both (i) diagnostic tests to spot patients most predisposed and in need to be helped or affected by a new medication, and (ii) a targeted therapy built on the test results. Genomics, proteomics, bioinformatics, and functional genomics are molecular biology technologies indispensable for the molecular theragnostics evolution. These technologies engender the genetic and protein data essential for the expansion of diagnostic examinations. Theragnostics comprises an extensive range of subjects, including pharmacogenomics, personalized remedies, and molecular imaging to produce effective novel targeted therapies with acceptable benefit/risk to patients and an improved molecular understanding to optimize medicine selection. Moreover, theragnostics can (i) monitor the treatment response, (ii) augment drug efficacy and security, and (iii) abolish superfluous or wrong patients' therapies. The final result is a significant cost reduction on behalf of the healthcare system. Nevertheless, introducing theragnostics tests into routine healthcare entails both a cost-effectiveness analysis and the readiness of proper and accessible testing systems $[18,20]$. The usage of smart devices, biosensors, and bio-actuators will:

- Decrease the occurrence of serious incidents;

- Improve the efficiency of therapies;

- Expand the QoE for patients and professionals;

- Shrink the number of patient admissions, sick days absences, and outpatient inspections; and

- Advance e-documentation and personal risk analysis.

\section{Discussion}

Numerous studies have pointed out that telemedicine will improve healthcare quality for patients. Several telemedicine applications show the potential CPSs for healthcare access improving quality, and efficiency in healthcare and telemedicine.

However, a key factor is how to design such systems using the CPS paradigm. Nevertheless, many organizations, healthcare centers, governmental agencies, private medical institutions, and medical professionals still need to discuss and assimilate the technological challenges involved in telemedicine systems. Furthermore, it is paramount to consider a privacy policy regarding patients' data due to the delicate nature of medical/healthcare information. 
Future developments will analyze solutions from other places and how they can be translated into Healthcare CPSs [2, 3, 4, 35]. Moreover, provisions for intelligent information retrieval and database handling must be thought [24, 25]. The most reasonable line of attack is to extend the Industry 4.0 standard to the healthcare domain (the so-called Health 4.0). This paradigm shift will permit healthcare CPSs to be compliant with other automated frameworks.

\section{Conclusions}

There is profuse evidence that Health 4.0 should borrow concepts from the Industry 4.0 standard. Due to the very definition of healthcare infrastructures as critical regarding security, robustness, and resilience, additional design principles for Health 4.0 have to be recognized as obligatory. Mobile devices can become future gateways for intelligent healthcare despite their presentday restricted communication routing ability, limited battery life, and best effort paradigm. Mobile gadgets must be compliant with smart healthcare via smart devices and general custom-made medicine methodologies (missioncritical QoS, multi-tenancy, and high QoE). However, smart devices and wearables will impact the transmission and reception of multimedia files and reports. Smart devices, biosensors, and bio-actuators will benefit from 5G technologies including NB- IoT will be available soon, which will prompt innovative business models. The healthcare industry might swing from the manufacturing to the service business by embracing new duties.

\section{Conflict of interest statement}

We certify that there is no conflict of interest with any financial organization in the subject matter or materials discussed in this manuscript.

\section{Authors' biography}

\section{A. C. B. Monteiro}

M.Sc. in Electrical Engineering from the State University of Campinas in telecommunications and signal processing. Ph.D. Candidate at Department of Communication (DECOM) da Universidade Estadual de Campinas (UNICAMP).

\section{Reinaldo P. França}

M.Sc. in Electrical Engineering from State University of Campinas in biomedical engineering and image processing. $\mathrm{PhD}$ Candidate at Departament of Communication (DECOM) da Universidade Estadual de Campinas (UNICAMP).

\section{Vania V. Estrela}

B.Sc. degree from Federal University of Rio de Janeiro (UFRJ) in Electrical and Computer Engineering (ECE); M.Sc. in ECE from the Technological Institute of Aeronautics (ITA) and Northwestern University, USA; and Ph.D. in ECE from the Illinois Institute of Technology (IIT), Chicago, IL, USA. Taught at: DeVry University; State University of Northern Rio de Janeiro (UENF), Rio de Janeiro (RJ), Brazil; and for the West Zone State University (UEZO), RJ. Research interests include signal/image/video processing, inverse problems, computational

\& mathematical modeling, stochastic models, multimedia, electronic instrumentation, machine learning and remote sensing. Reviewer for: 
IMAVIS (Elsevier); Pattern Recognition (Elsevier); COMPELECENG (Elsevier); Measurement (Elsevier); IET Image Processing; EURASIP Journal on Advances in Signal Processing (JASP) (Springer); IEEE Journal of Biomedical and Health

Informatics (JBHI); Int'1 J. of Electrical and Comp. Engineering (IJECE); Int'1

Journal of Ambient Computing and Intelligence (IJACI); Journal of Microwaves, Optoelectronics and Electromagnetic Applications (JMOE); and SET Int'l J. Broadcast Eng. (SET-IJBE). Engaged in topics such as technology transfer, STEM education, environmental issues and digital inclusion. Member of IEEE, and ACM. Editor of IJACI, EURASIP JASP, and SETIJBE.

\section{Yuzo Iano}

B.Sc. by the State University of Campinas/SP/Brazil-Unicamp in Electrical Engineering (1972), M.Sc. in Electrical Engineering from State University of Campinas (1974) and doctorate at Electrical Engineering from the same university (1986). He is currently full professor at Unicamp. Has experience in Electrical Engineering, focusing on Telecommunications, Electronics and Information Technology. $\mathrm{He}$ is working in the following subjects: digital transmission and processing of images/audio/video/data, HDTV, digital television, networks 4G/5G, middleware, transmission, canalization, broadcasting of television signals, pattern recognition, digital coding of signals, data transmission and storage, and smart/digital cities.

Dr Abdeldjalil Khelassi: is an Associate Professor at Tlemcen University, Algeria. He obtained his Doctor in Science (2013), Magister (2008) and Engineer (2004) in Computer Sciences from the Department of Computer Science at Tlemcen University. His research interest includes cognitive systems, knowledge-based systems, case-based reasoning, distributed reasoning, fuzzy sets theory and health science. He is the editor manager of Medical Technologies Journal and the co-editor in chief of Electronic Physician Journal.

\section{Navid Razmjooy}

B.Sc. by the Ardabil branch of IAU University/Iran (2007), M.Sc. from the Isfahan branch of IAU University/Iran with honor in Mechatronics Engineering (2011), Ph.D. in Control Engineering (Electrical Engineering) from by the Tafresh University/Iran (2018). Born born in 1988. Research opportunity at the Amirkabir University of Technology (2017-2018). He is working in the following subjects: Control, interval analysis, Optimization, Image Processing, Machine Vision, Soft Computing, Data Mining, Evolutionary Algorithms, and System Control. He is a senior member of IEEE/USA and YRC in the IAU/Iran. He published more than 75 papers and 3 books in English and Farsi in peer-reviewed journals and conferences and is now a reviewer in the national and international journals and conferences.

\section{References}

[1] Hermann, M., Pentek, T., \& Otto, B. (2016). Design Principles for Industrie 4.0 Scenarios. 2016 49th Hawaii International Conference on System Sciences (HICSS), 39283937.

[2] Lee EA (2008) Cyber-physical systems: design challenges. In 11th IEEE Symposium on Object and Component-Oriented Real-Time Distributed Computing (ISORC), pp 363-369. doi:10.1109/ISORC.2008.25 
[3] Estrela, V.V., Saotome, O., Loschi, H.J., Hemanth, J., Farfan, W.S., Aroma, J., Saravanan, C., \& Grata, E.G.H. (2018). Emergency response cyber-physical framework for landslide avoidance with sustainable electronics. Technologies, 6, 42. https://doi.org/10.3390/technologies6020042

[4] Estrela, V.V., Hemanth J., Saotome O., Grata, E.G.H., \& Izario, D.R.F. (2019). Emergency response cyber-physical system for flood prevention with sustainable electronics. In: Iano Y., Arthur R., Saotome O., Vieira Estrela V., Loschi H. (eds), In Proceedings of the 3rd Brazilian Technology Symposium. BTSym 2017. Springer, Cham.

[5] Acatech-National Academy of Science and Engineering (ed) (2011) Cyber-physical systems: driving force for innovation in mobility, health, energy and production. http:// www.acatech.de/fileadmin/user_upload/Baumstruktur_nach_Website/Acatech/root/de/Publi kation en/Stellungnahmen/acatech_POSITION_CPS_Englisch_WEB.pdf.Accessed 1 June 2018

[6] Vermesan O, Friess P (eds) (2015) Building the hyperconnected society: IoT research and innovation value chains, ecosystems and markets. River Publishers, Denmark

[7] European Commission (2012) eHealth Action Plan 2012-2020: Innovative healthcare for the 21st Century. https://ec.europa.eu/digital-single-market/en/news/ehealth-action-plan20122020-innovative-healthcare-21st-century.Accessed 1 June 2016

[8] Kagermann H, Wahlster W, Helbig J (eds) (2013) Recommendations for implementing the strategic initiative Industrie 4.0: Final report of the Industrie 4.0 Working Group. http://www.

acatech.de/fileadmin/user_upload/Baumstruktur_nach_Website/Acatech/root/de/Material_f uer_So nderseiten/Industrie_4.0/Final_report_Industrie_4.0_accessible.pdf. Accessed 1 June 2018

[9] NetNames (2015) Counting the cost of counterfeiting. https://www.netnames.com/assets/ shared/whitepaper/pdf/NetNames-Counterfeiting-Report-A4-2015.pdf. Accessed 1 June 2016 8. Fricker SA, Thümmler C, Gavras A (eds) (2015) Requirements engineering for digital health. Springer, Switzerland

[10] Van Rensburg, A. (2007). The value chain as an operations reference model, Philippine Industrial Engineering Journal, 4 (1).

[11] Thuemmler C., Chunxue Bai C. Health 4.0: Application of Industry 4.0 Design Principles in Future Asthma Management, Health 4.0: How Virtualization and Big Data are Revolutionizing

Healthcare, Chapter 2, Thuemmler, C.; Bai, C. (Eds) 2017, XII, 254, p. 78 ISBN: 978-3-3194761

[12]Needham C, Glasby, J (2015) Personalization-Love it or hate it. J Integr Care 23(5):268-

276

[13]European Commission (DG CONNECT) and China Academy of Information and Communication Technology (2016) EU-China Joint White Paper on the Internet of Things. https://ec.europa.eu/digital-single-market/en/news/eu-china-joint-white-paper-internetthings.

Accessed 1 July 2018

[14]European Commission, Information Society and Media Directorate-General (2011) Report on the public consultation on eHealth action plan 2012-2020

[15]Collins FS, Varmus H (2015) A new initiative on precision medicine. N Engl JMed 372:793-

795

[16]European Commission (2014) Digital Single Market, mHealth, Infographics. https://ec. europa.eu/digital-single-market/en/news/mhealth-what-it-infographic. Accessed 15 June 2018 15. Elias J (2015) In 2016, users will trust health apps more than their doctors. http://www.forbes.com/sites/jenniferelias/2015/12/31/in-2016-users-will-trust-health-appsmore-than-theirdoctors/\#7fd18292d5f6.Accessed 15 June 2018

[17]National Information Board, NHS England (2015) Personalised health and care 2020, $\mathrm{HM}$

Government https://www.gov.uk/government/uploads/system/uploads/attachment_data/file/ 384650/NIB_Report.pdf. Accessed 20 June 2018

[18]Jeelani S, Jagat Reddy RC, Maheswaran T, Asokan GS, Dany A, Anand B (2014) Theragnostics: a treasured tailor for tomorrow. J Pharm Bioallied Sci 6(Suppl 1): S6-S8. doi:10.4103/0975-7406.137249 
[19]Thuemmler C, Mival O, Benyon D et al (2013) Norms and standards in modular medical architectures. In: IEEE HealthCom 2013, conference publication

[20]Helfand C (2015) GSK jumps on the 'smart inhaler' bandwagon with Ellipta sensor deal. http://www.fiercepharma.com/regulatory/gsk-jumps-on-smart-inhaler-bandwagon-

elliptasensor-deal. Accessed 1 Jul 2018

[21]Boulet LP, Vervloet D, Magar Y, Foster JM (2012) Adherence: the goal to control asthma. Clin Chest Med 33(3):405-417

[22]Ceselli A, Premoli M, Secci S. Mobile Edge Cloud Network Design Optimization. IEEE/ACM Transactions on Networking, 2017 DOI: 10.1109/TNET.2017.2652850

[23]Tutac, A.E., Racoceanu, D., Leow, W., Müller, H., Putti, T.C., \& Cretu, V. (2009). Toward translational incremental similarity-based reasoning in breast cancer grading. Medical Imaging: Computer-Aided Diagnosis.

[24]Herrmann, A. E. \& Estrela, V. V. (2016). Content-based image retrieval (CBIR) in remote clinical diagnosis and healthcare. In M. Cruz-Cunha, I. Miranda, R. Martinho, \& R. Rijo (Eds.), Encyclopedia of E-Health and Telemedicine (pp. 495-520). Hershey, PA: IGI Global. doi:10.4018/978-1-4666-9978-6.ch039

[25]Jesus, M.A., \& Estrela, V.V. (2017). An Introduction to Data Mining Applied to HealthOriented Databases.Orient. J. Comp. Sci. and Technol (OJCST), 9(3). DOI: 10.13005/ojcst/09.03.03

[26]Jordao, K.; I. Yuzo; Loschi, Hermes José; Ferreira, L. A. S; Bianchini, D. Smart City IoT model: a prospective analysis considering indicators of social, technical and operational aspects. Int'l Conference on Information Society and Smart Cities (ISC 2018), 2018. ISC Conference Proceedings ISBN: 978-1-912532-02-5.

[27]Loschi HJ, Leon, J., Lustosa, T.C. ; Iano, Y., Duenas, A., Makluf, C. A., Camargo, E. R. M., Carvalho, S. Moretti, A.E.R. Cardoso, P. What Will 5G Be? Trends for nanotechnology, video systems, networks management, radio frequency, spectrum management, and regulation in Brazil. In: Brazilian Technology Symposium, 2015, Campinas. Proc. of Brazilian Technology Symposium, 2015. v. 1. p. 1-4. ISSN: 2447-8326

[28]Seger, W. "The Rediscovery of the Social Side of Medicine: Philosophy and Value of the

International Classification of Functioning, Disability and Health (ICF)". Medical Technologies Journal, Vol. 1, no. 1, Mar. 2017, pp. 3-3, doi:https://doi.org/10.26415/2572004X-vol1iss1p3-3.

[29]Belgherbi, A., I. Hadjidj, and A. Bessaid. "Computer-Aided Detection of Simultaneous Abdominal Organ from CT Images Based on Iterative Watershed Transform". Medical Technologies Journal, Vol. 1, no. 1, Mar. 2017, pp. 8-8, doi:https://doi.org/10.26415/2572004X-vol1iss1p8-8.

[30]de Jesus, M.A., Estrela, V.V., Saotome, O., \& Stutz, D. (2018) Super-resolution via particle swarm optimization variants. In: Hemanth J., Balas V. (Eds). Lecture Notes in Computational Vision and Biomechanics, vol 25. Springer, Cham.

[31]Fernandes, S.R., Estrela, V.V., Magalhaes, H.A., \& Saotome, O. (2014) On improving sub-pixel accuracy by means of B-Spline, In Proceedings of the 2014 IEEE international conference on imaging system and techniques (IST 2014), Santorini, Greece. doi: 10.1109/IST.2014.6958448

[32]Hemanth, D.J., \& Estrela, V.V. (2017). Deep Learning for Image Processing Applications, Advances in Parallel Computing Series, Vol. 31, IOS Press, ISBN 978-161499-821-1 (print), ISBN 978-1-61499-822-8 (online)

[33]Iano, Y. ; T. Lima, I. ; J. Loschi, H. ; C. Lustosa, T. ; S. Mesquita, O. ; Moretti, A. Sustainable Computing and Communications - Internet Broadband Network of Things Applied to Intelligent

Education. In Proceedings of the 4th Int'l Conf, on Smart Cities and Green ICT Systems, 2015,

Lisbon ISBN 978-989-758-105-2. 4th Int'l Conf. on Smart Cities and Green ICT Systems, 2015. p. 350-356. http://dx.doi.org/10.5220/0005447303500356

[34]Ebnehoseini, Z. "Health Information Search Engine the New Tools of Health Literacy". Medical Technologies Journal, Vol. 1, no. 4, Nov. 2017, pp. 107-, doi:https://doi.org/10.26415/2572-004X-vol1iss4p107-107.

[35]Temmar, R., M. Ouslim, and M. Mekri. "The New Design of a Remote Real Time Embedded Medical Platform". Medical Technologies Journal, Vol. 1, no. 3, Sept. 2017, pp. 55-56, doi:https://doi.org/10.26415/2572-004X-vol1iss3p55-56. 
Medical Technologies Journal, Volume: 2, Issue: 4, January-March 2018, Pages: 262-276. Doi : https://doi.org/10.26415/2572-004X-vol2iss1p262-276

[36]Mahmoodi, M. S., and S. A. Mahmoodi. "Design of CAD System of Solitary Pulmonary Nodule with Harmony Classification and Fuzzy System". Medical Technologies Journal, Vol. 1, no. 4, Nov. 2017, pp. 102-, doi:https://doi.org/10.26415/2572-004X-vol1iss4p102102.

[37]Razmjooy, N., B. S. Mousavi, Khalilpour, M. and Hosseini, H. "Automatic Selection and Fusion of Color Spaces for Image Thresholding”. Signal, Image and Video Processing, Vol. 8, no. 4, 2014: 603-614.

[38]Razmjooy, N., Mousavi B. S., Fazlollah S., Hosseini Khotbesara, M. "A ComputerAided Diagnosis System for Malignant Melanomas." Neural Computing and Applications, Vol. 23, no.

7-8, 2013: 2059-2071.

[39]Mousavi, B. S., and Soleymani, F., Razmjooy, F. "Semantic Image Classification by Genetic Algorithm Using Optimised Fuzzy System Based on Zernike Moments.” Signal, Image and Video Processing, Vol. 8, no. 5, 2014: 831-842. 